\title{
Coulisses
}

Revue de théâtre

13 | Hiver 1996

Varia

\section{Pour qu'on se souvienne du théâtre que faisait Jean-Luc Lagarce.}

\section{Pierre Frantz}

\section{(2) OpenEdition}

1 Journals

Édition électronique

URL : http://journals.openedition.org/coulisses/3921

DOI : 10.4000/coulisses.3921

ISSN : 2546-9460

Éditeur

Presses universitaires de Franche-Comté

\section{Édition imprimée}

Date de publication : 1 janvier 1996

Pagination : 4-6

ISSN : 1150-594X

\section{Référence électronique}

Pierre Frantz, «Pour qu'on se souvienne du théâtre que faisait Jean-Luc Lagarce. », Coulisses [En ligne], 13 | Hiver 1996, mis en ligne le 15 mars 2019, consulté le 05 octobre 2020. URL : http:// journals.openedition.org/coulisses/3921; DOI : https://doi.org/10.4000/coulisses.3921

Ce document a été généré automatiquement le 5 octobre 2020.

Coulisses 


\title{
Pour qu'on se souvienne du théâtre que faisait Jean-Luc Lagarce. ${ }^{1}$
}

\author{
Pierre Frantz
}

\section{Conversation avec}

Bernard Bloch,

un jour de novembre 
1 Propos recueillis par Pierre Frantz

On se coucherait pour toujours, on dirait qu'on est malade...

2 Bernard Bloch a joué le rôle d'Argan dans Le Malade imaginaire qu'a monté Jean-Luc Lagarce en $1993^{2}$. Ce spectacle a eu 150 représentations, en France et dans l'est de l'Allemagne.

PIERRE FRANTZ : Qu'est-ce que c'était, pour toi de jouer le malade dans la mise en scène de Lagarce ?

Bernard Bloch: C'est l'histoire d'une inversion permanente entre la maladie et la santé; entre le malade et le médecin; entre le metteur en scène et l'acteur. Qui était le médecin de l'autre?

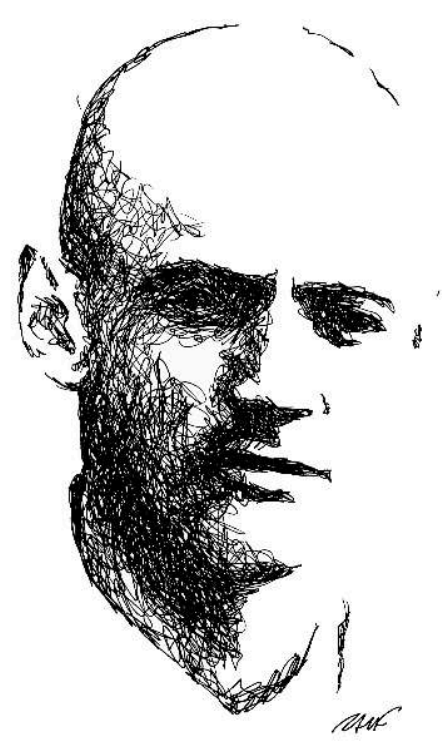
Selon les moments chacun était le médecin de l'autre. Enfin, dans le vrai sens du mot : un médecin c'est quelqu'un qui aide à vivre.

PF : Comment as-tu rencontré Argan et Jean-Luc Lagarce?

BB : C'était en juin 1992. On avait annulé le tournage d'un film dans lequel je devais jouer. J'avais du temps devant moi et je suis venu à Paris, voir des films, des spectacles et rencontrer des amis. L'un d'eux était malade. Je suis allé voir à la Cité Universitaire Les solitaires intempestifs, un spectacle écrit et mis en scène par Jean-Luc. Il était alors hospitalisé, atteint déjà par la maladie. Jean-Luc m'a fait demander si j'accepterais de jouer Argan. Je l'ai appelé à l'hôpital pour dire que oui et ne l'ai vu vraiment qu'à l'automne suivant.

Je n'avais jamais joué Molière. Mais, dans ma vie personnelle, j'avais compris depuis peu ce que signifiait jouer, se jouer la maladie. L'hypocondrie. On a répété cinq semaines. J'ai été surpris de trouver cela si difficile. La langue de Molière dans Le Malade est étonnamment résistante. Jean-Luc était là-dessus d'une extrême rigueur. Avec un sens calviniste du travail. J'ai lu, pendant les répétitions, un de ses textes : un type vient rendre visite à sa famille avec l'intention de lui annoncer sa maladie. À la fin de la pièce, il n'a pas parlé mais tout entendu de ce que chacun lui a dit.

PF : Comment les répétitions se sont-elles passées ? Intervenait-il beaucoup?

BB : Non, il était peu interventionniste. Il laissait à l'acteur son autonomie. Mais il avait une vision dramaturgique forte. Parfois, et il demandait qu'on l'en excuse, il préférait regarder le spectacle à partir des coulisses. Moi, j'aimais le savoir là et, quand je quittais le plateau, (Argan sort très peu de scène) il prenait mon bâton. Je soufflais alors un peu près de lui. Je suis devenu très ami avec lui, par ces moments là, partagés dans la coulisse.

PF : Est-ce qu'il parlait de sa maladie?

BB : Il était très discret. Juste une anecdote de temps en temps. Un jour, il a dû faire la queue pendant quatre heures à la sécurité sociale. Pour prouver qu'il n'était pas mort. On est pris en charge à $100 \%$ par la Sécu quand on a le Sida ; mais seulement de quatre mois en quatre mois... 
PF : Pourquoi Le malade imaginaire?

BB : Monter cette pièce, ça a été une incroyable traversée de la maladie. Du concept même de maladie. L'hypocondrie c'est une maladie imaginaire. Une maladie de l'imaginaire. La maladie d'Argan devient une forte expression de vitalité. Jean-Luc tenait donc à rompre avec toute tradition d'une interprétation du rôle comme celui d'un vieillard débile. Il a voulu un Argan dans la force de l'âge face à un Béralde vraiment malade. La mort est autour d'Argan. Il se fait bébé, fils de sa femme, prêt à se débarrasser de ses autres enfants. Argan fuit la mort en remontant vers cet autre néant, celui qui est avant la naissance, d'où l'on vient et où on est bien.

PF : Comment Jean-Luc Lagarce réagissait-il à cette pièce ?

BB : Il riait beaucoup de cette «farce en grand deuil» comme a dit un critique. Il avait vraiment un regard gai. Un enfant, lui a demandé, à Montbéliard, je crois, «à quoi on voit que c'est la dernière pièce de Molière?» Et il a répondu que Molière ignorait que ce serait sa dernière pièce. Il n'y avait au reste rien de dépressif chez Jean-Luc, ni pour lui, ni pour les autres. C'était quelqu'un qui aimait la vie, qui aimait manger solidement ; au propre comme au figuré.

$\mathbf{P F}$ : Et toi, comment as-tu vécu ce rôle?

BB : C'était très dur. Au bout d'une vingtaine de représentations, je me suis mis à haïr ce personnage d'Argan. C'était très dur mais ça produisait du jeu, par toute cette matérialité qu'on investit quand on joue et qui n'est pas du côté du sens. C'est un peu l'expérience du Paradoxe de Diderot. Après, au moment de la troisième série de représentations le bonheur du jeu est revenu. Il se trouve que j'avais aussi retrouvé ma parole de metteur en scène.

PF : Dans Le malade imaginaire, comment avez-vous vu, Jean-Luc Lagarce et toi, les rapports du théâtre, de la théâtralité, et de cette thématique si particulière?

BB : D'un côté, Argan, c'est vraiment une complexion d'acteur. Quelqu'un qui manipule et se fait manipuler. Une marionnette qui est aussi, dans cette maladie imaginaire, la marionnette de lui- même.

Et puis, comme disait quelque part Jean-Loup Rivière, le théâtre vient combler un besoin de croyance. Ce serait une religion mais une religion libre, qui commence au début de la représentation, à laquelle on adhère librement et qui cesse quand le rideau tombe. Argan meurt et ressuscite. Comme la petite Louison. On joue à voir comment c'est après. C'était le rôle de Molière et Molière revit aussi chaque fois qu'on le joue. Au reste Molière, "ce bon impertinent" de Molière qui fait des comédies, est nommé dans la pièce, comme si chaque fois il était encore vivant.

\section{NOTES}

1. Depuis le début de sa publication, en Avril 90, Coulisses suit le travail de J.L. Lagarce : $\mathrm{n}^{\circ} 1$ L'Itinéraire d'un jeune auteur; p. 27 - n 5 La Cantatrice chauve; p. 6 - n 9 Dossier Molière, Le Malade imaginaire, p. $41-\mathrm{n}^{\circ} 10$ L'T̂le des esclaves, p. $28-\mathrm{n}^{\circ} 12$ La Cagnotte, p. 56 
2. Le Malade imaginaire, mise en scène Jean-Luc Lagarce, Décor Laurent Peduzzi, avec Bernard Bloch, Mireille Herbstmeyer, Irina Dalle, Sylvie Faivre, Jean-Louis Grinfeld, Olivier Py, Philippe Lehembre, Olivier Achard, François Berreur, Elisabeth Mazev. Coproduction Théâtre de la Roulotte, Théâtre Granit. 